\title{
On the minimum rainbow subgraph number of a graph
}

\author{
Ingo Schiermeyer \\ Institut für Diskrete Mathematik und Algebra, Technische Universität Bergakademie \\ Freiberg, 09596 Freiberg, Germany
}

Received 19 October 2011, accepted 9 April 2012, published online 1 June 2012

\begin{abstract}
We consider the Minimum Rainbow SUbGRAPH problem (MRS): Given a graph $G$ whose edges are coloured with $p$ colours. Find a subgraph $F \subseteq G$ of minimum order and with $p$ edges such that each colour occurs exactly once. This problem is NP-hard and APX-hard.

For a given graph $G$ and an edge colouring $c$ with $p$ colours we define the rainbow subgraph number $r s(G, c)$ to be the order of a minimum rainbow subgraph of $G$ with size $p$. In this paper we will show lower and upper bounds for the rainbow subgraph number of a graph.
\end{abstract}

Keywords: Edge colouring, rainbow subgraph.

Math. Subj. Class.: 05C15, 05C35

\section{Introduction and motivation}

We use [2] for terminology and notation not defined here and consider finite and simple graphs only.

Our research was motivated by the following problem from bioinformatics. The problem data consist in a set $\mathcal{G}$ of $p$ genotypes $g_{1}, g_{2}, \ldots, g_{p}$ corresponding to $p$ individuals in a population. Each genotype $g$ is a vector with entries in $\{0,1,2\}$. Each position where a 2 appears is called ambiguous position. For a genotype $g$ we have to determine a pair of haplotypes $h_{P}$ and $h_{M}$ ( $h_{P}$ stands for the paternal haplotype and $h_{M}$ stands for the maternal haplotype), which are binary vectors such that $g=h_{P} \oplus h_{M}$.

Given two haplotypes $h^{\prime}$ and $h^{\prime \prime}$, their sum is defined as the vector $g=h^{\prime} \oplus h^{\prime \prime}$ with $g[i]=0$, if $h^{\prime}[i]=h^{\prime \prime}[i]=0, g[i]=1$, if $h^{\prime}[i]=h^{\prime \prime}[i]=1$ and $g[i]=2$, if $h^{\prime}[i] \neq h^{\prime \prime}[i]$.

We say that a set $\mathcal{H}$ of haplotypes resolves $\mathcal{G}$ if for every $g \in \mathcal{G}$ there exist $h_{1}, h_{2} \in \mathcal{H}$ such that $g=h_{1} \oplus h_{2}$. Given a set $\mathcal{G}$ of genotypes, the haplotyping problem consists

E-mail address: Ingo.Schiermeyer@tu-freiberg.de (Ingo Schiermeyer) 
in finding a set $\mathcal{H}$ of haplotypes that resolves $\mathcal{G}$. In the Pure Parsimony Haplotyping problem (PPH problem) we are interested in finding a set $\mathcal{H}$ of smallest possible cardinality. If each genotype has at most $k$ ambiguous positions, then we denote this problem by $\operatorname{PPH}(k)$. The PPH problem has been studied in ([3],[4],[7],[9]).

Matos Camacho et al. [8] have shown that the $\mathrm{PPH}(k)$ can be transformed to a graph problem, the Minimum RAINBOW SUBGRAPH problem (MRS). Note that this edge-colouring need not be proper.

Definition 1.1 (Rainbow subgraph).

Let $G$ be a graph with an edge-colouring. A subgraph $H$ of $G$ is called rainbow subgraph if $H$ does not contain two edges of the same colour.

Definition 1.2 (Minimum Rainbow Subgraph problem (MRS)).

Given a graph $G$, whose edges are coloured with $p$ colours, find a subgraph $F \subseteq G$ of minimum order and with $p$ edges such that each colour occurs exactly once.

For a set $\mathcal{G}$ of $p$ genotypes $g_{1}, g_{2}, \ldots, g_{p}$ we will use $p$ colours $1,2, \ldots, p$. For each haplotype we introduce a vertex. If two haplotypes $h^{\prime}$ and $h^{\prime \prime}$ resolve a genotype $g_{i}\left(g_{i}=\right.$ $\left.h^{\prime} \oplus h^{\prime \prime}\right)$, then the corresponding vertices will be joined by an edge which receives colour $i$. If a genotype is resolved by two identical haplotypes, then the corresponding vertex is joined by an edge which is called a loop.

In this way we construct a graph $G$, whose edges are coloured with $p$ colours. Note that this is a proper edge colouring (no vertex is incident with two edges of the same colour), since a haplotype $h$ can be used at most once in a pair of haplotypes, which resolves a genotype $g$. Furthermore, every set $\mathcal{H}$ of haplotypes that resolves $\mathcal{G}$ corresponds to a rainbow subgraph $F$ of $G$.

It has been shown in [8] that a graph $G$ containing loops can be transformed into a graph $G^{\prime}$ without loops. Hence in the following we may assume that all graphs have no loops.

Matos Camacho et al. [8] proved the MRS problem to be NP-hard and APX-hard. In [5] it has been shown that the MRS problem remains NP-hard and APX-hard even for graphs with maximum degree 2 .

Remark: If we do not consider edge colourings, the analogous problem is known as the $(t, f(t))$ dense subgraph problem $((t, f(t))$-DSP), which asks whether there is a $t$-vertex subgraph of a given graph $G$ which has at least $f(t)$ edges. When $f(t)=\left(\begin{array}{l}t \\ 2\end{array}\right),(t, f(t))$-DSP is equivalent to the well-known $t$-clique problem (cf. [1]).

\section{Lower bounds for the rainbow subgraph number}

Definition 2.1. Let $G$ be a graph and $c$ be its edge colouring with $p$ colours. The rainbow subgraph number of $G$ (with respect to the colouring $c$ ) is defined as the order of its minimum rainbow subgraph of size $p$, and denoted by $\operatorname{rs}(G, c)$ (or $r s(G)$, when the colouring $c$ is clear from the context).

Improved lower bounds for the rainbow subgraph number $r s(G)$ will be of major importance for the design of approximation algorithms with better approximation ratios for the MRS problem (cf. [8,5]). So far nothing better than the trivial lower bound $r s(G) \geq \frac{2 p}{\Delta(G)}$ is known. We can improve this lower bound by counting the number of distinct colours among all edges incident to a vertex. 
Definition 2.2. Given an edge colouring of a graph $G$ with colours $1,2, \ldots, p$, we define $c(e)=i$, if the edge $e$ has colour $i$ for $1 \leq i \leq p$.

Let $c d(v)$ (colour degree) denote the number of distinct colours among all edges incident to the vertex $v$ and let $c d(i)=\max \{c d(v) \mid v \in V(G)$ has an incident edge with colour $i\}$ be the maximum colour degree for every colour $i, 1 \leq i \leq p$.

Using the maximum colour degrees for all colours we can show the following improved lower bound.

Proposition 2.3. Let $G$ be a graph, whose edges are coloured with p colours. Then

$$
r s(G) \geq \sum_{i=1}^{p} \frac{2}{c d(i)} \geq \frac{2 p}{\Delta(G)} .
$$

Proof. Let $F$ be a minimum rainbow subgraph of order $k=r s(G)$. Then

$$
r s(G)=k=\sum_{v \in V(F)} \frac{d_{F}(v)}{d_{F}(v)}=\sum_{e=u w, e \in E(F)} \frac{1}{d_{F}(u)}+\frac{1}{d_{F}(w)} \geq \sum_{i=1}^{p} \frac{2}{c d(i)} \geq \frac{2 p}{\Delta(G)} .
$$

The following example shows that this bound is sharp and improves the lower bound of $\frac{2 p}{\Delta(G)}$ significantly.

Example 2.4. For $p \geq 4$ and $\Delta \geq 2$ let $G=K_{1, \Delta}+C_{p-1}$ (where $G+H$ denotes the disjoint union of two graphs $G$ and $H$ ). All edges of the cycle $C_{p-1}$ are coloured distinctly, say with colours $1,2, \ldots, p-1$, and all edges of $K_{1, \Delta}$ are coloured with colour $p$. Then $r s(G)=p+1=p-1+2=\sum_{i=1}^{p} \frac{2}{c d(i)}>\frac{2 p}{\Delta(G)}$.

We can further improve this lower bound by counting the number of distinct colours among all edges incident to the endvertices of an edge. For this purpose we define $q(i)=$ $\min \left\{\frac{1}{c d(u)}+\frac{1}{c d(w)} \mid u w \in E(G)\right.$ and $\left.c(u w)=i\right\}$.

Proposition 2.5. Let $G$ be a graph, whose edges are coloured with p colours. Then

$$
r s(G) \geq \sum_{i=1}^{p} q(i) \geq \sum_{i=1}^{p} \frac{2}{c d(i)} \geq \frac{2 p}{\Delta(G)}
$$

Proof. Let $F$ be a minimum rainbow subgraph of order $k=r s(G)$. For every colour $i, 1 \leq$ $i \leq p$, let $u_{i} w_{i}$ be an edge such that $\frac{1}{c d\left(u_{i}\right)}+\frac{1}{c d\left(w_{i}\right)}=q(i)$. If $u w \in E(F)$ is an edge with $c(u w)=i$, then $\frac{1}{c d(u)}+\frac{1}{c d(w)} \geq \frac{1}{c d\left(u_{i}\right)}+\frac{1}{c d\left(w_{i}\right)}=q(i) \geq 2 \cdot \frac{1}{\max \left\{c d\left(u_{i}\right), c d\left(w_{i}\right)\right\}} \geq \frac{2}{c d(i)}$. Therefore,

$$
\begin{aligned}
& r s(G)=k=\sum_{v \in V(F)} \frac{d_{F}(v)}{d_{F}(v)}=\sum_{e=u w, e \in E(F)} \frac{1}{d_{F}(u)}+\frac{1}{d_{F}(w)} \geq \\
& \sum_{i=1}^{p} q(i) \geq \sum_{i=1}^{p} \frac{2}{c d(i)} \geq \frac{2 p}{\Delta(G)} .
\end{aligned}
$$


The following example shows that this bound is sharp and improves the previous two lower bounds significantly.

Example 2.6. Let $G \cong K_{1, p}$ for some $p \geq 2$. Let the edges of $G$ be coloured with $p$ colours. Then $c d(i)=p$ and $q(i)=1+\frac{1}{p}$ for $1 \leq i \leq p$. Thus $\operatorname{rs}(G)=p+1=$ $p \cdot\left(1+\frac{1}{p}\right)=\sum_{i=1}^{p} q(i)>2=p \cdot \frac{2}{p}=\sum_{i=1}^{p} \frac{2}{c d(i)}=\frac{2 p}{\Delta\left(K_{1, p}\right)}$.

\section{Upper bounds for the rainbow subgraph number}

First observe that the trivial upper bound $r s(G) \leq 2 p$ is achieved if the rainbow subgraph $F$ is a matching. This upper bound has been improved towards $r s(G) \leq 2 p+1-\Delta(G)$ by Koch [6] for properly edge-coloured graphs and this bound is sharp. For instance, let $G=K_{1, \Delta}+(p-\Delta) K_{2}$, where $p \geq \Delta$, and all edges of $G$ are coloured distinctly. Then $r s(G)=2 p+1-\Delta(G)$.

Similar to Brooks' Theorem (cf. [2]) we can characterize all graphs achieving this bound.

Theorem 3.1. Let $G$ be a graph with maximum degree $\Delta \geq 2$, whose edges are properly coloured with $p$ colours. If $r(G)=2 p+1-\Delta(G)$, then $G$ has the following properties:

1. G contains a star $K_{1, \Delta}$ with center vertex $v_{0}$ and leaves $v_{1}, \ldots, v_{\Delta}$ and $G\left[N\left(v_{0}\right)\right]$ is edgeless. Let $c\left(v_{0} v_{i}\right)=i$ for $1 \leq i \leq \Delta$ and $H_{0} \cong G\left[N\left[v_{0}\right]\right]$.

2. If $p>\Delta$, then let $H_{i}$ be the subgraph spanned by the edges with colour $i$ for $\Delta+$ $1 \leq i \leq p$. The subgraphs $H_{\Delta+1}, H_{\Delta+2}, \ldots, H_{p}$ are pairwise vertex-disjoint and $V\left(H_{0}\right) \cap V\left(H_{i}\right)=\emptyset$ for $\Delta+1 \leq i \leq p$.

3. $E\left(H_{i}, H_{j}\right)=\emptyset$ for $\Delta+1 \leq i<j \leq p$ (where $E\left(H_{i}, H_{j}\right)$ is the set of all edges having one vertex in $V\left(H_{i}\right)$ and the other vertex in $V\left(H_{j}\right)$ ).

4. $E\left(v_{i}, H_{j}\right)=\emptyset$ for $1 \leq i \leq \Delta$ and $\Delta+1 \leq j \leq p$ (where $E\left(v_{i}, H_{j}\right)$ is the set of all edges incident with $v_{i}$ and $a$ vertex in $V\left(H_{j}\right)$ ).

5. If $u v \in E\left(H_{i}\right)$ for some $\Delta+1 \leq i \leq p$, then $N(u) \cap N(v)=\emptyset$.

6. $N\left(v_{i}\right) \cap N\left(v_{j}\right)=\emptyset$ for $v_{i} \in V\left(H_{i}\right), v_{j} \in V\left(H_{j}\right), \Delta+1 \leq i<j \leq p$.

Proof. 1. Suppose there is an edge $v_{i} v_{j}$ for some $1 \leq i<j \leq \Delta$. If $c\left(v_{i} v_{j}\right)=k$ for some $k$ with $1 \leq k \leq \Delta, k \neq i, j$, then $r s(G) \leq(\Delta+1)-1+(2 p-2 \Delta)=2 p-\Delta<$ $2 p+1-\Delta$, a contradiction. If $c\left(v_{i} v_{j}\right)=k$ for some $k$ with $\Delta+1 \leq k \leq p$, then $r s(G) \leq(\Delta+1)+(2 p-2 \Delta-2)=2 p-\Delta-1<2 p+1-\Delta$, a contradiction as well.

2. Suppose there are integers $i, j$ with $\Delta+1 \leq i<j \leq p$ and two adjacent edges $e, f$ with $c(e)=i, c(f)=j$. Then $r s(G) \leq(\Delta+1)+(2 p-2 \Delta-1)=2 p-$ $\Delta<2 p+1-\Delta$, a contradiction. Suppose there are integers $i, j$ with $1 \leq i \leq$ $\Delta, \Delta+1 \leq j \leq p$ and two adjacent edges $e, f$ with $c(e)=i, c(f)=j$. Then $r s(G) \leq(\Delta+1)+(2 p-2 \Delta-1)=2 p-\Delta<2 p+1-\Delta$, a contradiction as well.

3. Suppose there is an edge $v_{i} v_{j}$ with $v_{i} \in V\left(H_{i}\right), v_{j} \in V\left(H_{j}\right), \Delta+1 \leq i<j \leq p$. Then $c\left(v_{i} v_{j}\right)=k$ for some $1 \leq k \leq \Delta$. Hence $r s(G) \leq(\Delta+1)-1+(2 p-2 \Delta)=$ $2 p-\Delta<2 p+1-\Delta$, a contradiction.

4. Suppose there is an edge $v_{i} v_{j}$ for two vertices $v_{i} \in V\left(H_{0}\right)$ and $v_{j} \in V\left(H_{j}\right), \Delta+1 \leq$ $j \leq p$. Then $r s(G) \leq(\Delta+1)+(2 p-2 \Delta-1)=2 p-\Delta<2 p+1-\Delta$, a contradiction. 
5. Suppose there is an edge $u v \in E\left(H_{i}\right)$ for some $\Delta+1 \leq i \leq p$ with $N(u) \cap N(v) \neq$ $\emptyset$. By 3. and 4. we conclude that $N(u) \cap N(v) \cap V\left(H_{0}\right)=\emptyset$. Furthermore, for a vertex $w \in N(u) \cap N(v)$, we have $c(u w)=j, c(v w)=k$ for some $1 \leq j<k \leq \Delta$. Then $r s(G) \leq(\Delta+1)-2+(2 p-2 \Delta+1)=2 p-\Delta<2 p+1-\Delta$, a contradiction.

6. Suppose $N\left(v_{i}\right) \cap N\left(v_{j}\right) \neq \emptyset$ for two vertices $v_{i} \in V\left(H_{i}\right), v_{j} \in V\left(H_{j}\right), \Delta+1 \leq i<$ $j \leq p$. By 3. and 4. we conclude that $N\left(v_{i}\right) \cap N\left(v_{j}\right) \cap V\left(H_{0}\right)=\emptyset$. Furthermore, for a vertex $w \in N\left(v_{i}\right) \cap N\left(v_{j}\right)$, we have $c(u w)=k, c(v w)=l$ for some $1 \leq k<l \leq \Delta$. Then $r s(G) \leq(\Delta+1)-2+(2 p-2 \Delta+1)=2 p-\Delta<2 p+1-\Delta$, a contradiction.

Another upper bound for the rainbow subgraph number follows from an approach presented in [8]. Observe that two adjacent edges of different colours together have three vertices, whereas two edges of different colours in a matching have four vertices. Based on this obervation the following algorithm has been proposed in [8].

\section{Algorithm}

Input: A graph $G$ of order $n$ whose edges are coloured with $p$ colours

1. Construct a graph $G^{\prime}$ with $V\left(G^{\prime}\right)=\left\{v_{1}, v_{2}, \ldots, v_{p}\right\}\left(v_{i}\right.$ corresponds to colour $\left.i\right)$ and $v_{i} v_{j} \in E\left(G^{\prime}\right)$ if there exist two adjacent edges $e, f \in E(G)$ with $c(e)=i$ and $c(f)=j(c(x)$ denotes the colour of the edge $x)$.

2. Now compute a maximum matching $M$ of order $\beta\left(G^{\prime}\right)$ in $G^{\prime}$. This can be done in polynomial time.

3. Next construct a graph $H$ with $V(H) \subseteq V(G)$ as follows: For each matching edge of $M$ choose two adjacent edges in $G$ with these two colours. For each vertex of $V\left(G^{\prime}\right)$ not in $M$ choose an edge in $G$ with this colour. In this way we obtain a rainbow subgraph $H \subseteq G$ with $|E(H)|=p$.

Correctness of the algorithm: Edges of the matching correspond to pairs of adjacent edges in the original graph. Colours that are left out by this procedure are added greedily at the end.

Claim 3.2. $|V(H)| \leq 2 p-\beta\left(G^{\prime}\right)$

Proof. For each matching edge of $G^{\prime}$ three vertices appear in $H$. Hence

$$
|V(H)| \leq 3 \beta\left(G^{\prime}\right)+2\left(p-2 \beta\left(G^{\prime}\right)\right)=2 p-\beta\left(G^{\prime}\right)
$$

Corollary 3.3. $r s(G) \leq 2 p-\beta\left(G^{\prime}\right)$.

\section{Acknowledgement}

We thank the referees for some valuable comments. 


\section{References}

[1] Y. Asahiro, R. Hassin and K. Iwama, Complexity of finding dense subgraphs, Discrete Appl. Math. 121 (2002), 15-26.

[2] J. A. Bondy and U.S.R. Murty, Graph Theory, Springer, London, 2008.

[3] D. Catanzaro and M. Labbé, The pure parsimony haplotyping problem: overview and computational advances, International Transactions in Operational Research 16 (2009), 561-584.

[4] D. Gusfield, Haplotype inference by pure Parsimony, in: Proceedings ot the 14th annual conference on Combinatorial pattern matching (CPM'03), Springer, Berlin, Heidelberg, 2003, 144155.

[5] J. Katrenič and I. Schiermeyer, Improved approximation bounds for the minimum rainbow subgraph problem, Inf. Process. Lett. 111 (2011), 110-114.

[6] M. Koch, Das Population-Haplotyping-Problem: Graphentheoretische Ansätze, Diploma thesis, TU Bergakademie Freiberg, 2008.

[7] G. Lancia, M. C. Pinotti and R. Rizzi, Haplotyping Populations by Pure Parsimony: Complexity of Exact and Approximation Algorithms, INFORMS Journal on Computing 16 (2004), 348-359.

[8] S. Matos Camacho, I. Schiermeyer and Z. Tuza. Approximation algorithms for the minimum rainbow subgraph problem, Discrete Math. 310 (2010), 2666-2670.

[9] L. S. Wang, Hapolotype inference by maximum parsimony, Bioinformatics 19 (2003), $1773-$ 1780. 